\title{
Bit Commitment from Weak Non-Locality
}

\author{
Stefan Wolf \\ Département d'Informatique et R.O. \\ Université de Montréal, Canada \\ Email: wolf@iro.umontreal.ca
}

\author{
Jürg Wullschleger \\ Département d'Informatique et R.O. \\ Université de Montréal, Canada \\ Email: wullschj@iro.umontreal.ca
}

\begin{abstract}
So-called non-local boxes, which have been introduced as an idealization-in different respects-of the behavior of entangled quantum states, have been known to allow for unconditional bit commitment between the two involved parties. We show that, actually, any possible non-local correlation which produces random bits on both sides can be used to implement bit commitment, and that this holds even when the parties are allowed to delay their inputs to the box. Since a particular example is the behavior of an EPR pair, this resource allows for implementing unconditionally secure bit commitment as long as the parties cannot entangle their Qbits with any other system.
\end{abstract}

\section{INTRODUCTION AND PRELIMINARIES}

\section{A. Previous Work and Our Result}

Since cryptographic functionalities often cannot be realized in an unconditionally secure way from scratch, it is an interesting problem to find simple and weak information-theoretic primitives from which they can be realized. A particular class of such underlying primitives are those which stem from quantum physics. For instance, Bennett and Brassard have shown that two parties can generate a common secret key in an unconditionally secure way if they are connected by a quantum channel [1].

Another central result in this context by Mayers [11] states that it is impossible to realize bit commitment in an unconditionally secure way for both parties, even when they are connected by a quantum channel. It is important to note that the latter result even holds when the parties share a given pure state, for instance an EPR pair,

$$
\frac{1}{\sqrt{2}}(|00\rangle+|11\rangle)
$$

initially. Roughly speaking, an attacker can entangle his part of the state. Actually, it is a consequence of our results that every attack requires to use such entanglement: Bit commitment from a shared EPR pair is possible as soon as the parties cannot entangle their respective systems with any other system.

In order to get a better understanding of quantum-physical phenomena such as entanglement or non-locality, the behavior of quantum states has often been modeled as "boxes," i.e., conditional probability distributions characterizing the joint input-output behavior of the two- (or more) partite system. A particular box that has been well-studied recently [3], [4], [5], [6], [9], [13], [14], [10] is the so-called non-local box [12], or NL box for short, the behavior of which does actually not correspond to the behavior of any quantum state, but is an idealization thereof. (The NL box is, however, also "non-signaling," i.e., its behavior does not allow for message transmission). It has been shown in [15] that such an NL box is essentially equivalent to oblivious transfer: A single realization of one primitive perfectly allows for realizing the other. Interestingly, this fact implies that oblivious transfer is, as the NL box, symmetric, i.e., its direction can be perfectly inverted for free.

Since oblivious transfer allows for bit commitment, this result seems to contradict Mayers' impossibility theorem. It does not, however, since an NL box is not a quantum state, and it is a natural question what the decisive difference is. In [13], it has been suggested that it is the fact that in the quantum setting, a party can delay her measurement. In [5] it was shown, however, that NL boxes which do allow such a delay as well can nevertheless be used to implement bit commitment. Another potential reason is that the non-locality of the NL box is "superstrong," i.e., stronger than the one of any quantum state. In this paper, we show that this is not the case either: Even weak non-local behaviors do the job, for instance the one arising from EPR pairs. We can, therefore, conclude that the crucial point is that in the quantum setting, a party can entangle her system with another, but not in the case of a box. In other words, such states do actually allow for bit commitment as long as the parties cannot entangle their system with any other.

\section{B. Definitions and Preliminaries}

Definition 1: A bit commitment scheme is a pair of protocols Commit and Open executed between two parties, $A$ and $B$. First, Commit is executed, where $A$ has an input $v$ and $B$ has no input. $B$ can either accept or reject the execution of Commit. Then, Open is executed, where $B$ has an output $v^{\prime}$. $B$ either accepts or rejects the execution of Open. The two protocols must have the following properties:

- Correctness. If both parties are honest, then $B$ should always accept, with $v^{\prime}=v$.

- Privacy. If $A$ is honest, then the execution of Commit does not reveal any information about $v$ to $B$.

- Binding. If $B$ is honest and accepts after the execution of Commit, then there exists only one value $v^{\prime}$ (which is equal to $v$ if $A$ is honest) that $B$ accepts as output after the execution of Open.

Definition 2: A non-signaling box (NS box for short) is a box to which Alice can input a value $X$ and Bob a value 
$Y$. Alice then gets a value $A \in\{0,1\}$ and Bob gets a value $B \in\{0,1\}$ such that $\operatorname{Pr}[A=a, B=b \mid X=x, Y=y]=$ $P_{A B \mid X Y}(a, b, x, y)$. (Here, we assume that a party receives its output immediately after giving her input, independently of whether the other has already given his input or not. Note that the non-signaling condition implies that this is possible.) Furthermore, the following conditions must hold:

- Non-signaling. For all values $i, x, y \in\{0,1\}$, we have

$$
\begin{aligned}
& \operatorname{Pr}[A=i \mid X=x, Y=y]=1 / 2, \\
& \operatorname{Pr}[B=i \mid X=x, Y=y]=1 / 2 .
\end{aligned}
$$

- Dependence. $P_{A B \mid X Y} \neq P_{A \mid X} P_{B \mid Y}$.

Note that the non-signaling condition means that the output of one player is independent of the input of the other, thus the box does not allow for message transmission. If we had $P_{A B \mid X Y}=P_{A \mid X} P_{B \mid Y}$, the box would just consist of two local channels. This is excluded by the second condition, but the dependence can be arbitrarily weak. In particular, it may be some correlation that can be simulated using an EPR pair. The above-mentioned NL box is a special case of a non-signaling box, where we have $P_{A B \mid X Y}(a, b, x, y)=1 / 2$ if $a \oplus b=x y$ and 0 otherwise.

We now introduce two technical lemmas used later.

Lemma 1 (Chernoff): Let $X_{1}, X_{2}, \ldots, X_{n}$ be independent random variables with $\operatorname{Pr}\left[X_{i}=1\right]=p$ and $\operatorname{Pr}\left[X_{i}=0\right]=$ $1-p$. Let $X=\sum_{i=1}^{n} X_{i}$. For any $t \geq 0$ we have

$$
\begin{aligned}
& \operatorname{Pr}[X \geq E[X]+t] \leq e^{-2 t^{2} / n} \\
& \operatorname{Pr}[X \geq E[X]-t] \geq e^{-2 t^{2} / n} .
\end{aligned}
$$

Lemma 2: For any $n$ we have

$$
\sum_{i=0}^{r}\left(\begin{array}{l}
n \\
i
\end{array}\right) \leq 2^{n / 2+2 r-2 r^{2} / n} \leq 2^{n / 2+2 r} .
$$

Proof: ${ }^{i=0}$ Let $X_{1}, X_{2}, \ldots, X_{n}$ be independent random variables with $\operatorname{Pr}\left[X_{i}=1\right]=1 / 2$ and $\operatorname{Pr}\left[X_{i}=0\right]=1 / 2$. Let $X=\sum_{i=1}^{n} X_{i}$. We have

$$
\operatorname{Pr}[X \leq r]=\sum_{i=0}^{r}\left(\begin{array}{c}
n \\
i
\end{array}\right) 2^{-n} .
$$

Using the Chernoff inequality, setting $r=n / 2-t$, we get

$$
\operatorname{Pr}[X \leq r] \leq e^{-2 t^{2} / n} \leq 2^{-n / 2+2 r-2 r^{2} / n},
$$

and therefore

$$
\sum_{i=0}^{r}\left(\begin{array}{l}
n \\
i
\end{array}\right) \leq 2^{n} 2^{-n / 2+2 r-2 r^{2} / n} \leq 2^{n / 2+2 r} .
$$

\section{Bit COMMITMENT FROM ANY NON-SignALING BOX}

In this section, we show how to realize unconditionally secure bit commitment from any NS box. Let $n$ be the number of calls to the NS box. Let $s=n^{3 / 4}$ be a security parameter, let $k_{1}=n^{3 / 4}, k_{2}=8 k_{1}+2 s, m=n / 2+4 k_{1}+s$, and $d=2 k_{1}+k_{2}+1$. Let, finally, $l>0$ and $k \geq m+2 s+l$. Let $\mathcal{C} \subset$ $\{0,1\}^{n}$ be a $(n, k, d)$-linear code, i.e., with $2^{k}$ elements and minimal distance $d$. Since we do not have to decode $\mathcal{C}$, we can use a random linear code. If $k \leq(1-H(d / n)) n-s$ a random code has a minimal distance of at least $d$ with probability at least $1-2^{-s}$. Since $k=l+n / 2+o(n)$ and $d / n=o(1)$, we can choose $l=n / 2-o(n)$. Let $h:\{0,1\}^{*} \times\{0,1\}^{n} \rightarrow\{0,1\}^{m}$ and ext : $\{0,1\}^{*} \times\{0,1\}^{n} \rightarrow\{0,1\}^{l}$ be universial hash functions. Let $v \in\{0,1\}^{l}$.

Protocol 1: Commit $(v)$.

- Alice chooses $x \in_{R} \mathcal{C}$, Bob chooses $y \in_{R}\{0,1\}^{n}$.

- Alice and Bob input $x$ and $y$ component-wise to the NS box. Alice gets $a \in\{0,1\}^{n}$ and Bob gets $b \in\{0,1\}^{n}$.

- Bob chooses $r_{1} \in_{R}\{0,1\}^{*}$ and sends it to Alice.

- Alice sends Bob $h\left(r_{1}, a\right)$.

- Alice chooses $r_{2} \in_{R}\{0,1\}^{*}$ and sends $\left(r_{2}, v \oplus\right.$ $\left.\operatorname{ext}\left(r_{2}, x\right)\right)$ to Bob.

Protocol 2: Open().

- Alice sends Bob $x, a$, and $v$.

- Bob checks whether $x \in \mathcal{C}$ holds, $h\left(r_{1}, a\right)$ is correct, whether the sequence $\left(a_{i}, b_{i}, x_{i}, y_{i}\right)$ has the right statistics, i.e., is distributed according to $P_{A B X Y}=$ $P_{X} P_{Y} P_{A B \mid X Y}$, and whether $v \oplus \operatorname{ext}\left(r_{2}, x\right)$ is correct. If all these checks are $o k$, he accepts and outputs $v$. Otherwise, he rejects.

In the following we will show that these two protocols implement bit commitment, i.e., that it satisfies the three conditions correctness, privacy, and binding.

Lemma 3: The protocols Commit and Open satisfy the correctness condition with an error negligible in $n$.

Proof: Bob always accepts Commit. If Alice follows the protocols, then $h(x)$ and $v \oplus \operatorname{ext}\left(r_{2}, x\right)$ will be correct and $x \in \mathcal{C}$ holds. Furthermore, with overwhelming probability, the sequence $\left(a_{i}, b_{i}, x_{i}, y_{i}\right)$ will have the right statistics. Therefore, Bob accepts Open with overwhelming probability and outputs $v$, the value Alice was commited to.

Bob cannot cheat actively since he does not send any message. The following lemma proves that he does not get any information if Alice is honest.

Lemma 4: The protocols Commit and Open satisfy the privacy condition with an error of at most $2^{-s}$.

Proof: Let us assume that Alice is honest. We will show that with probability at least $1-2^{-s}$, Bob does not get any information about $v$ before the opening. Since the box is nonsignaling, Bob's values $y$ and $b$ are independent of $x$. Since Alice chooses $x$ uniformely, its min-entropy is equal to $k$. The additional randomness $r_{2}$ is independent of $x$, so all the information Bob gets about $x$ is $h\left(r_{1}, x\right)$, which has length $m$. Therefore, Bob's min-entropy about $x$ is at least $k-m$. It follows from the leftover hash lemma [8], [2], [7] that extracting $l=k-m-2 s$ bits makes the key uniform with an error of at most $2^{-s}$. So Bob does not get any information about $v$ with probability at least $1-2^{-s}$.

It remains to be shown that the protocols are binding. Without loss of generality, we can assume that Alice will finally give some input to all the boxes. Let her $i$-th input be $x_{i}$, and let her outcome be $a_{i}$. Of course, she is not supposed to send Bob the true values $x_{i}, a_{i}$, but she may change some 
of them. However, if she changes more than $k_{1}=n^{3 / 4}$ values $x_{i}$ or $a_{i}$, the sequence $\left(a_{i}, b_{i}, x_{i}, y_{i}\right)$ will have the correct statistics only with negligible probability. A malicious Alice may also choose not to give input values to some of the boxes until the opening phase. Let the number of these values be $k_{2}$.

Lemma 5: If Alice does not input any values to at least $k_{2}$ calls to the NS box, the probability that there exists a value $a^{\prime}$ that has a Hamming distance of at most $k_{1}$ from her final value $a$ such that $h\left(r_{1}, a^{\prime}\right)=h$ is at most $2^{-s+1}$.

Proof: Alice has to send a hash value $h$ before the opening phase. In the opening phase, she inputs the remaining $k_{2}$ values to the box and gets random outputs for them. So she gets randomly one out of $2^{k_{2}}$ possible values for $a$. She can freely choose $h$, so she may also choose a value $a_{h}$ such that $h\left(r_{1}, a_{h}\right)=h$. The probability that the Hamming distance between $a_{h}$ and $a$ is smaller than $k_{1}$ is at most

$$
\sum_{i=0}^{k_{1}}\left(\begin{array}{c}
k_{2} \\
i
\end{array}\right) 2^{-k_{2}} \leq 2^{-k_{2}+k_{2} / 2+4 k_{1}}=2^{-k_{2} / 2+4 k_{1}}=2^{-s} .
$$

For any value $a^{\prime} \neq a_{h}$, the probability that $h\left(r_{1}, a^{\prime}\right)=h$ is equal to $2^{-m}$. So the probability that there is another value $a^{\prime}$ with Hamming distance of at most $k_{1}$ near $a$, such that $h\left(r_{1}, a^{\prime}\right)=h$ is at most

$$
\sum_{i=0}^{k_{1}}\left(\begin{array}{c}
n \\
i
\end{array}\right) 2^{-m} \leq 2^{-m+n / 2+4 k_{1}}=2^{-s} .
$$

The statement follows. We have applied Lemma 2 twice.

In order to be able to open a commitment to two different values $v$ and $v^{\prime}$, Alice needs to find two strings $x$ and $x^{\prime}$ which are compatible with the commitment and such that her success probability is maximized. We will now show that under certain conditions, there never exist two values $x$ and $x^{\prime}$ such that Bob would accept the opening for both.

Lemma 6: If Alice changes only $k_{1}$ pairs and delays only $k_{2}$ inputs, then the protocol is binding as long as $2 k_{1}+k_{2}<d$.

Proof: Any two valid inputs strings $x$ and $x^{\prime}$ have a distance of at least $d$. If we ignore all the positions where Alice did not input anything to the box, $x$ and $x^{\prime}$ still have a distance of at least $d-k_{2}$ values. Only one $x^{\prime} \in \mathcal{C}$ is closer than $\left(d-k_{2}\right) / 2$ to the $x$ that Alice has chosen.

We are now able to prove the binding condition.

Lemma 7: The protocols Commit and Open satisfy the binding condition with an error negligible in $n$.

Proof: If Alice changes at least $k_{1}=n^{3 / 4}$ values, she has only exponentially small probability of success. Otherwise, if she does not input anything to at least $k_{2}$ calls to the box, she has a probability of success of at most $2^{-s+1}$. But otherwise, she will not be able to cheat, if $2 k_{1}+k_{2}<d$. Hence, her overall success probability is negligible.

Theorem 1: There exists a reduction of bit commitment to any NS box.

\section{CONCLUSIONS}

We have shown that unconditionally secure bit commitment between two parties can be obtained from any bi-partite "input-output box" which produces random bits on both sides, does not allow for signaling, and is not "separable" (i.e., consists of two independent channels on both sides). It is important to note that, as in [5], this result even holds when this box is such that each party can choose to delay certain inputs (without the other party being aware of this: the box will produce an output on the other side nevertheless). An example of behavior such a box can have is the one of an EPR pair under measurements. This result does not contradict Mayers' famous impossibility result since such boxes are not quantum, and do not allow the parties to entangle their parts of the system with another system, an operation-this is a consequence of our results-necessary to carry out a successful attack.

\section{ACKNOWLEDGEMENTS}

We thank Thomas Holenstein, Louis Salvail, and Christian Schaffner for helpful discussions.

\section{REFERENCES}

[1] C. H. Bennett, G. Brassard, Quantum Cryptography: Public Key Distribution and Coin Tossing Proceedings of IEEE International Conference on Computers Systems and Signal Processing, pp. 175-179, 1984.

[2] C. Bennett, G. Brassard, C. Crépeau, and U. Maurer, Generalized privacy amplification, IEEE Transactions on Information Theory, 41, 1995.

[3] A. Broadbent and A. A. Méthot, On the power of non-local boxes, quant-ph/0504136 2005.

[4] G. Brassard, H. Buhrman, N. Linden, A. A. Methot, A. Tapp, and F. Unger, A limit on nonlocality in any world in which communication complexity is not trivial, quant-ph/0508042 2005.

[5] H. Buhrman, M. Christandl, F. Unger, S. Wehner, A. Winter, Implications of superstrong nonlocality for cryptography, quant-ph/0504133 2005.

[6] N. J. Cerf, N. Gisin, S. Massar, and S. Popescu, Quantum entanglement can be simulated without communication, quant-ph/0410027 2004.

[7] J. Håstad, R. Impagliazzo, L. A. Levin, and M. Luby. A pseudorandom generator from any one-way function. SIAM Journal on Computing, 28(4):1364-1396, 1999.

[8] R. Impagliazzo, L. A. Levin, and M. Luby. Pseudo-random generation from one-way functions. In Proc. 21st ACM Symp. on Theory of Computing, pages 12-24. ACM, 1989.

[9] N. S. Jones and L. Masanes, Interconversion of nonlocal correlations, quant-ph/0506182 2005.

[10] L. Masanes, A. Acin, and N. Gisin, General properties of nonsignaling theories, quant-ph/0508016 2005.

[11] D. Mayers, Unconditionally secure quantum bit commitment is impossible, Phys. Rev. Lett., Vol. 78, pp. 3414-3417, 1997.

[12] S. Popescu and D. Rohrlich, Causality and nonlocality as axioms for quantum mechanics, quant-ph/9709026 1997.

[13] S. Short, N. Gisin, and S. Popescu, The physics of no-bitcommitment: generalized quantum non-locality versus oblivious transfer, quant-ph/0504134 2005.

[14] S. Short, N. Gisin, and S. Popescu, Entanglement swapping for generalized non-local correlations, quant-ph/0508120 2005.

[15] S. Wolf and J. Wullschleger, Oblivious transfer and quantum nonlocality, Proceedings of the IEEE International Symposium on Information Theory (ISIT '05), 2005. 